Open J. Math. Sci., Vol. 1(2017), No. 1, pp. 25 - 33

Website: https://pisrt.org/psr-press/journals/oms/

ISSN: 2523-0212 (Online) 2616-4906 (Print)

http://dx.doi.org/10.30538/oms2017.0003

\title{
SOME MULTI-STEP ITERATIVE METHODS FOR SOLVING NONLINEAR EQUATIONS-I
}

\author{
MUHAMMAD SAQIB ${ }^{1}$, MUHAMMAD IQBAL
}

\begin{abstract}
In this paper, we suggest and analyze two new algorithm of fourth and fifth order convergence. We rewrite nonlinear equation as an equivalent coupled system and then use modified decomposition technique to develop our algorithms. Convergence analysis of newly introduced algorithms has been discussed. To see efficiency and performance of these algorithms, we have made comparison of these algorithms with some well known algorithms existing in literature.
\end{abstract}

AMS Mathematics Subject Classification: 39B12, 34A34.

Key words and phrases: iterative methods; nonlinear system; convergence.

\section{Introduction}

To find the root of nonlinear equation of the form $f(x)=0$, is the oldest and basic problem in numerical analysis. Newton's method is one of oldest and most powerful formula to approximate the root of nonlinear equations. It has second order convergence. Many modifications in Newton's method have been made to increase its convergence order using various techniques. Recently, many iterative methods with higher order convergence have been established using different techniques like Taylor's series, Adomain decomposition, homotopy, modified homotopy, decomposition, modified decomposition, interpolation, quadrature rules and their many modifications. see $[1,2,3,4,5,6,7,8,9,10,11]$ and references therein. Chun [3] introduced some multi-step iterative methods using Adomain decomposition. These method requires higher order derivatives. Later on, Noor

Received 08 July 2017 . Revised 10 September 2017.

1 Corresponding Author

(C) 2017 Muhammad Saqib, Muhammad Iqbal. This is an open access article distributed under the Creative Commons Attribution License, which permits unrestricted use, distribution, and reproduction in any medium, provided the original work is properly cited. 
$[4,5]$ established some multi-step iterative methods that do not require higher order derivative using a different technique.

In this paper, some numerical methods based on decomposition technique are proposed for solving algebraic nonlinear equations. For this purpose, we write nonlinear equations as an equivalent system of equations and use technique introduced by Gejji and Jafari [8]. Recently, several iterative methods have been suggested by writing nonlinear equations as an equivalent system of equations and then using different techniques. In section 3, we give detailed proof regarding convergence of our newly established iterative methods. In the last section, numerical results are given to make comparison of these algorithm with some classical methods.

\section{Iterative methods}

Consider the nonlinear equation

$$
f(x)=0 ; \quad x \in \mathbb{R},
$$

assume $\alpha$ is a simple root of (1) and $\gamma$ is an initial guess sufficiently close to $\alpha$. We can write (1) as following coupled system;

$$
\begin{gathered}
f(\gamma)+(x-\gamma) f^{\prime}\left(\frac{x+\gamma}{2}\right)+g(x)=0, \\
g(x)=f(x)-f(\gamma)-(x-\gamma) f^{\prime}\left(\frac{x+\gamma}{2}\right) .
\end{gathered}
$$

From Eq. (2), we have

$$
x=\gamma-\frac{f(\gamma)}{f^{\prime}\left(\frac{x+\gamma}{2}\right)}-\frac{g(x)}{f^{\prime}\left(\frac{x+\gamma}{2}\right)} .
$$

Let

$$
x=c+N(x),
$$

where

$$
c=\gamma-\frac{f(\gamma)}{f^{\prime}\left(\frac{x+\gamma}{2}\right)},
$$

and

$$
N(x)=-\frac{g(x)}{f^{\prime}\left(\frac{x+\gamma}{2}\right)} .
$$

Now, we construct a sequence of higher-order iterative methods by applying modified decomposition technique introduced by Gejji and Jafari [8]. This technique consists a solution of equation (3) that can be written in the form of infinite series:

$$
x=\sum_{i=0}^{\infty} x_{i},
$$


and using Gejji and Jafari [8] technique, we decompose the nonlinear function $N$ as;

$$
N\left(\sum_{i=0}^{\infty} x_{i}\right)=N\left(x_{0}\right)-\sum_{i=1}^{\infty}\left\{N\left(\sum_{j=0}^{i} x_{j}\right)-N\left(\sum_{j=0}^{i-1} x_{j}\right)\right\} .
$$

Thus from Eqs. (4), (7) and (8) we have

$$
\sum_{i=0}^{\infty} x_{i}=x_{0}+N\left(x_{0}\right)+\sum_{i=1}^{\infty}\left\{N\left(\sum_{j=0}^{i} x_{j}\right)-N\left(\sum_{j=0}^{i-1} x_{j}\right)\right\} .
$$

So we have iteration scheme as

$$
\begin{aligned}
x_{0}= & c \\
x_{1}= & N\left(x_{0}\right) \\
x_{2}= & N\left(x_{0}+x_{1}\right)-N\left(x_{0}\right) \\
& \cdot \\
& \cdot \\
& \cdot \\
x_{n+1}= & N\left(x_{0}+x_{1}+\ldots+x_{n}\right)-N\left(x_{0}+x_{1}+\ldots+x_{n-1}\right), n=1,2, \ldots
\end{aligned}
$$

When

$$
\begin{aligned}
x & \approx x_{0} \\
& =c \\
& =\gamma-\frac{f(\gamma)}{f^{\prime}\left(\frac{x+\gamma}{2}\right)} .
\end{aligned}
$$

From above relation, we can write the algorithm as follows;

Algorithm 2.1: For any initial value $x_{0}$, we compute the approximation solution $x_{n+1}$, by the iteration scheme;

$$
\begin{aligned}
y_{n} & =x_{n}-\frac{f\left(x_{n}\right)}{f^{\prime}\left(x_{n}\right)} \\
x_{n+1} & =x_{n}-\frac{f\left(x_{n}\right)}{f^{\prime}\left(\frac{x_{n}+y_{n}}{2}\right)} .
\end{aligned}
$$

This algorithm has third order convergence and has been established by Frontini and Sormani [1].

When

$$
\begin{aligned}
x & \approx x_{0}+x_{1} \\
& =\gamma-\frac{f(\gamma)}{f^{\prime}\left(\frac{x+\gamma}{2}\right)}-\frac{g\left(x_{0}\right)}{f^{\prime}\left(\frac{x+\gamma}{2}\right)} .
\end{aligned}
$$


From Eq. (3), we see that

$$
g\left(x_{0}\right)=f\left(x_{0}\right)
$$

by substituting in above, we get

$$
x=\gamma-\frac{f(\gamma)}{f^{\prime}\left(\frac{x+\gamma}{2}\right)}-\frac{f\left(x_{0}\right)}{f^{\prime}\left(\frac{x_{0}+\gamma}{2}\right)},
$$

Using this, we suggest the following three step iterative methods for solving Eq. (1) as follows;

Algorithm 2.2: For any initial value $x_{0}$, we compute the approximation solution $x_{n+1}$, by the iteration scheme;

$$
\begin{array}{ll}
\text { Predictor Steps; } & y_{n}=x_{n}-\frac{f\left(x_{n}\right)}{f^{\prime}\left(x_{n}\right)} \\
z_{n}= & x_{n}-\frac{f\left(x_{n}\right)}{f^{\prime}\left(\frac{x_{n}+y_{n}}{2}\right)} \\
\text { Corrector Step; } & x_{n+1}=z_{n}-\frac{f\left(z_{n}\right)}{f^{\prime}\left(\frac{x_{n}+z_{n}}{2}\right)}
\end{array}
$$

When

$$
\begin{aligned}
x & \approx x_{0}+x_{1}+x_{2} \\
& =x_{0}+N\left(x_{0}+x_{1}\right) \\
& =x_{0}-\frac{g\left(x_{0}+x_{1}\right)}{f^{\prime}\left(\frac{x_{0}+x_{1}+\gamma}{2}\right)} \\
& =x_{0}-\frac{f\left(x_{0}+x_{1}\right)+f\left(x_{0}\right)}{f^{\prime}\left(\frac{x_{0}+x_{1}+\gamma}{2}\right)}
\end{aligned}
$$

From this relation, we formulate four step iterative method for solving Eq. (1) as follows;

Algorithm 2.3: For any initial value $x_{0}$, we compute the approximation solution $x_{n+1}$, by the iteration scheme;

$$
\begin{aligned}
\text { Predictor Steps; } & y_{n}=x_{n}-\frac{f\left(x_{n}\right)}{f^{\prime}\left(x_{n}\right)} \\
z_{n} & =x_{n}-\frac{f\left(x_{n}\right)}{f^{\prime}\left(\frac{x_{n}+y_{n}}{2}\right)} \\
u_{n} & =z_{n}-\frac{f\left(z_{n}\right)}{f^{\prime}\left(\frac{x_{n}+z_{n}}{2}\right)} \\
\text { Corrector Step; } & x_{n+1}=z_{n}-\frac{f\left(u_{n}\right)+f\left(z_{n}\right)}{f^{\prime}\left(\frac{x_{n}+u_{n}}{2}\right)}
\end{aligned}
$$




\section{Convergence Analysis}

In this section, we discuss in detail the convergence analysis of Algorithm 2.2 and 2.3 established above.

Theorem 3.1. For an open interval $I \subset \mathbb{R}$ let $f: I \rightarrow \mathbb{R}$ and $\alpha \in I$ be simple zero of $f(x)=0$. If $f$ is differentiable and $x_{0}$ is sufficiently close to $\alpha$ then threestep iterative method defined by Algorithm 2.2 has fourth order convergence.

Proof. Expanding $f(x)$ by Taylor's series about $\alpha$, we have

$$
f\left(x_{n}\right)=f^{\prime}(\alpha)\left(e_{n}+c_{2} e_{n}^{2}+c_{3} e_{n}^{3}+c_{4} e_{n}^{4}+c_{5} e_{n}^{5}+\ldots\right.
$$

where $c_{k}=\frac{1}{k !} \frac{f^{(k)}(\alpha)}{f^{\prime}(\alpha)}$, and $e_{n}=x_{n}-\alpha$.

$$
\begin{gathered}
f^{\prime}\left(x_{n}\right)=f^{\prime}(\alpha)\left(1+2 c_{2} e_{n}+3 c_{3} e_{n}^{2}+4 c_{4} e_{n}^{3}+5 c_{5} e_{n}^{4}+6 c_{6} e_{n}^{5}+\ldots\right. \\
y_{n}=x_{n}-\frac{f\left(x_{n}\right)}{f^{\prime}\left(x_{n}\right)}
\end{gathered}
$$

From above equations, we get

$$
\begin{aligned}
y_{n} & =\alpha+c_{2} e_{n}^{2}+\left(2 c_{3}-2 c_{2}^{2}\right) e_{n}^{3}+\left(3 c_{4}-7 c_{2} c_{3}+4 c_{2}^{3}\right) e_{n}^{4}+\left(4 c_{5}-10 c_{2} c_{4}\right. \\
& \left.-6 c_{3}^{2}+20 c_{3} c_{2}^{2}-8 c_{2}^{4}\right) e_{n}^{5}+\ldots
\end{aligned}
$$

Now, expanding $f^{\prime}\left(\frac{x_{n}+y_{n}}{2}\right)$ by Taylor's series about $\alpha$,

$$
\begin{aligned}
f^{\prime}\left(\frac{x_{n}+y_{n}}{2}\right) & =f^{\prime}(\alpha)\left\{1+c_{2} e_{n}+\left(c_{2}^{2}+\frac{3}{4} c_{3}\right) e_{n}^{2}+\left(\frac{7}{2} c_{2} c_{3}-2 c_{2}^{3}+\frac{1}{2} c_{4}\right) e_{n}^{3}\right. \\
& \left.+\left(3 c_{3}^{2}-\frac{37}{4} c_{3} c_{2}^{2}+\frac{3}{2} c_{2} c_{4}+4 c_{2}^{4}+\frac{5}{16} c_{5}\right) e_{n}^{4}+\ldots\right\}
\end{aligned}
$$

Dividing Eq. (9) by Eq. (12)

$$
\begin{aligned}
\frac{f\left(x_{n}\right)}{f^{\prime}\left(\frac{x_{n}+y_{n}}{2}\right)} & =e_{n}+\left(-c_{2}^{2}+\frac{1}{4} c_{3}\right) e_{n}^{3}+\left(-\frac{15}{4} c_{2} c_{3}+3 c_{2}^{3}+\frac{1}{2} c_{4}\right) e_{n}^{4} \\
& +\left(-\frac{51}{16} c_{3}^{2}+\frac{22}{7} c_{3} c_{2}^{2}-2 c_{2} c_{4}-6 c_{2}^{4}+\frac{16}{11} c_{5}\right) e_{n}^{5}+\ldots
\end{aligned}
$$

Now

$$
z_{n}=x_{n}-\frac{f\left(x_{n}\right)}{f^{\prime}\left(\frac{x_{n}+y_{n}}{2}\right)}
$$

Putting values in above, we get

$$
\begin{aligned}
z_{n} & =\alpha+\left(c_{2}^{2}-\frac{1}{4} c_{3}\right) e_{n}^{3}+\left(\frac{15}{4} c_{2} c_{3}-3 c_{2}^{3}-\frac{1}{2} c_{4}\right) e_{n}^{4} \\
& +\left(\frac{51}{16} c_{3}^{2}-\frac{22}{7} c_{3} c_{2}^{2}+2 c_{2} c_{4}-6 c_{2}^{4}+\frac{16}{11} c_{5}\right) e_{n}^{5}+\ldots
\end{aligned}
$$

Expanding $f\left(z_{n}\right)$ and $f^{\prime}\left(\frac{x_{n}+z_{n}}{2}\right)$ by Taylor's series about $\alpha$,

$$
f\left(z_{n}\right)=f^{\prime}(\alpha)\left\{\left(c_{2}^{2}-\frac{1}{4} c_{3}\right) e_{n}^{3}+\left(\frac{15}{4} c_{2} c_{3}-3 c_{2}^{3}-\frac{1}{2} c_{4}\right) e_{n}^{4}\right.
$$




$$
\begin{gathered}
\left.+\left(\frac{51}{16} c_{3}^{2}-\frac{22}{7} c_{3} c_{2}^{2}+2 c_{2} c_{4}-6 c_{2}^{4}+\frac{16}{11} c_{5}\right) e_{n}^{5}+\ldots\right\} \\
f^{\prime}\left(\frac{x_{n}+z_{n}}{2}\right)=f^{\prime}(\alpha)\left\{1+c_{2} e_{n}+\frac{3}{4} c_{3} e_{n}^{2}+\left(c_{2}^{3}-\frac{1}{4} c_{2} c_{3}+\frac{1}{2} c_{4}\right) e_{n}^{3}+\left(\frac{21}{4} c_{3} c_{2}^{2}-3 c_{2}^{3}\right.\right. \\
\left.\left.-\frac{1}{2} c_{2} c_{4}+\frac{5}{16} c_{5}-\frac{3}{8} c_{3}^{2}\right) e_{n}^{4}+\ldots\right\}
\end{gathered}
$$

Dividing (15) by Eq. (16), we have

$$
\begin{aligned}
\frac{f\left(z_{n}\right)}{f^{\prime}\left(\frac{x_{n}+z_{n}}{2}\right)} & =\left(c_{2}^{2}-\frac{1}{4} c_{3}\right) e_{n}^{3}+\left(4 c_{2} c_{3}-4 c_{2}^{3}-\frac{1}{2} c_{4}\right) e_{n}^{4}+\left(\frac{27}{8} c_{3}^{2}-\frac{73}{4} c_{3} c_{2}^{2}\right. \\
& \left.+\frac{5}{2} c_{2} c_{4}+10 c_{2}^{4}-\frac{11}{16} c_{5}\right) e_{n}^{5}+\ldots
\end{aligned}
$$

Corrector step of Algorithm 2.2 is

$$
x_{n+1}=z_{n}-\frac{f\left(z_{n}\right)}{f^{\prime}\left(\frac{x_{n}+z_{n}}{2}\right)}
$$

By substitution and simplification, we have

$$
x_{n+1}=\alpha+\left(4 c_{2} c_{3}-4 c_{2}^{3}-\frac{1}{2} c_{4}\right) e_{n}^{4}+\left(\frac{27}{8} c_{3}^{2}-\frac{73}{4} c_{3} c_{2}^{2}+\frac{5}{2} c_{2} c_{4}+10 c_{2}^{4}-\frac{11}{16} c_{5}\right) e_{n}^{5}+\ldots
$$

Hence Algorithm 2.2 has fourth order convergence.

Theorem 3.2. Let $f: I \subset \mathbb{R} \rightarrow \mathbb{R}$ for an open interval $I$ and $\alpha \in I$ be simple zero of $f(x)=0$. If $f$ is differentiable and $x_{0}$ is sufficiently close to $\alpha$ then three-step iterative method defined by Algorithm 2.3 has fifth order convergence and satisfies the error equation

$$
x_{n+1}=\alpha+\left(-\frac{1}{4} c_{3} c_{2}^{2}+c_{2}^{4}\right) e_{n}^{5}+O\left(e_{n}^{6}\right), \text { and } e_{n}=x_{n}-\alpha .
$$

Proof. From Eq. (17), we have

$$
u_{n}=\alpha+\left(4 c_{2} c_{3}-4 c_{2}^{3}-\frac{1}{2} c_{4}\right) e_{n}^{4}+\left(\frac{27}{8} c_{3}^{2}-\frac{73}{4} c_{3} c_{2}^{2}+\frac{5}{2} c_{2} c_{4}+10 c_{2}^{4}-\frac{11}{16} c_{5}\right) e_{n}^{5}+\ldots,
$$

Expanding $f\left(u_{n}\right)$ and $f^{\prime}\left(\frac{x_{n}+u_{n}}{2}\right)$ by Taylor's series about $\alpha$,

$$
\begin{gathered}
f\left(u_{n}\right)=f^{\prime}(\alpha)\left\{\left(4 c_{2} c_{3}-4 c_{2}^{3}-\frac{1}{2} c_{4}\right) e_{n}^{4}+\left(\frac{27}{8} c_{3}^{2}-\frac{73}{4} c_{3} c_{2}^{2}+\frac{5}{2} c_{2} c_{4}+10 c_{2}^{4}-\frac{11}{16} c_{5}\right) e_{n}^{5}+\ldots\right\}, \\
f^{\prime}\left(\frac{x_{n}+u_{n}}{2}\right)=f^{\prime}(\alpha)\left\{1+c_{2} e_{n}+\frac{3}{4} c_{3} e_{n}^{2}+\frac{1}{2} c_{4} e_{n}^{3}+\left(c_{2}^{3}-\frac{1}{4} c_{3} c_{2}^{2}+\frac{5}{16} c_{4}\right) e_{n}^{4}+\ldots\right\} .
\end{gathered}
$$

Now

$$
x_{n+1}=z_{n}-\frac{f\left(u_{n}\right)+f\left(z_{n}\right)}{f^{\prime}\left(\frac{x_{n}+u_{n}}{2}\right)},
$$

by substituting values and simplifying, we have

$$
x_{n+1}=\alpha+\left(-\frac{1}{4} c_{3} c_{2}^{2}+c_{2}^{4}\right) e_{n}^{5}+O\left(e_{n}^{6}\right) .
$$


Hence Algorithm 2.3 has fifth order convergence.

\section{Applications}

In this section, we present some numerical examples to examine the validity and efficieny of our newly devolped algorithms namely, Algorithm 2.2 and Algorithm 2.3. We also provide comparison of these algorithms with Newton's method(NM), Abbasbandy's method (AM), Householder's method(HHM), Halley's method (HM), Chun's method (CM) [3] and Noor's method(NR)[4]. We use $\epsilon=10^{-25}$. We use the following stopping criteria;

$$
\text { (ii) } \quad\left|f\left(x_{n}\right)\right|<\epsilon
$$

We consider the following nonlinear scalar equations for comparison:

$$
\begin{aligned}
& f_{1}(x)=\sin ^{2} x-x^{2}+1=0 \\
& f_{2}(x)=x^{2}-e^{x}-3 x+2=0 \\
& f_{3}(x)=\cos x-x=0 \\
& f_{4}(x)=(x-1)^{3}-1=0 \\
& f_{5}(x)=x^{3}-10=0 \\
& f_{6}(x)=e^{x^{2}+7 x-30}-1=0 .
\end{aligned}
$$

\begin{tabular}{|c|c|c|c|}
\hline \multicolumn{4}{|l|}{$\begin{array}{l}\text { Examples } \\
f_{1}, x_{0}=1\end{array}$} \\
\hline$N M$ & 7 & 1.40449164831534122635086 & $-1.05 e^{-50}$ \\
\hline$A M$ & 5 & 1.40449164831534122635086 & $-5.82 e^{-54}$ \\
\hline$H M$ & 4 & 1.40449164831534122635086 & $-5.45 e^{-63}$ \\
\hline$C M$ & 5 & 1.40449164831534122635086 & $-2.01 e^{-64}$ \\
\hline$H H M$ & 6 & 1.40449164821534122603508 & $1.82 e^{-25}$ \\
\hline$N R$ & 5 & 1.40449164821534122603508 & $9.23 e^{-26}$ \\
\hline Alg. 2.2 & 3 & 1.40449164821534122603508 & $3.40 e^{-25}$ \\
\hline Alg. 2.3 & 3 & 1.40449164821534122603509 & $2.32 e^{-44}$ \\
\hline \multicolumn{4}{|l|}{$f_{2}, x_{0}=2$} \\
\hline$N M$ & 6 & 0.257530285439860760455367 & $2.94 e^{-55}$ \\
\hline$A M$ & 5 & 0.257530285439860760455367 & $1.03 e^{-63}$ \\
\hline$H M$ & 5 & 0.257530285439860760455367 & 0 \\
\hline$C M$ & 4 & 0.257530285439860760455367 & $1.05 e^{-62}$ \\
\hline$H H M$ & 5 & 0.257530285439860760455367 & $-6.01 e^{-25}$ \\
\hline$N R$ & 5 & 0.257530285439860760455367 & $1.08 e^{-23}$ \\
\hline Alg. 2.2 & 3 & 0.257530285439860934975933 & $1.72 e^{-69}$ \\
\hline Alg.2.3 & 2 & 0.257530285439860934975933 & $1.01 e^{-29}$ \\
\hline
\end{tabular}

\section{Comparison Table}




\begin{tabular}{|c|c|c|c|}
\hline $\begin{array}{c}\text { Examples } \\
f_{3}, x_{0}=1.7\end{array}$ & Iterations & $x_{n}$ & $f\left(x_{n}\right)$ \\
\hline$N M$ & 5 & 0.739085133215160641655372 & $-2.04 e^{-31}$ \\
\hline$A M$ & 4 & 0.739085133215160641655372 & $-7.13 e^{-47}$ \\
\hline$H M$ & 4 & 0.739085133215160641655372 & $-5.04 e^{-58}$ \\
\hline$C M$ & 4 & 0.739085133215160641655372 & 0 \\
\hline$H H M$ & 4 & 0.739085133215160641655372 & $3.91 e^{-17}$ \\
\hline$N R$ & 4 & 0.739085133215160645628855 & $6.66 e^{-18}$ \\
\hline Alg. 2.2 & 3 & 0.739085133215160641655312 & $3.21 e^{-50}$ \\
\hline Alg. 2.3 & 3 & 0.739085133215160641655312 & $1.21 e^{-100}$ \\
\hline \multicolumn{4}{|l|}{$f_{4}, x_{0}=1.5$} \\
\hline$N M$ & 8 & 2.0000000000000000000001234 & $2.05 e^{-42}$ \\
\hline$A M$ & 5 & 2 & 0 \\
\hline$H M$ & 5 & 2 & 0 \\
\hline$C M$ & 5 & 2 & 0 \\
\hline$H H M$ & 7 & 2.000000000000000000000828 & $2.47 e^{-22}$ \\
\hline$N R$ & 5 & 2.000000000000000000000302 & $1.12 e^{-24}$ \\
\hline Alg. 2.2 & 4 & 2.000000000000000000000007 & $1.83 e^{-44}$ \\
\hline Alg. 2.3 & 4 & 2.000000000000000000000000 & $5.07 e^{-27}$ \\
\hline \multicolumn{4}{|l|}{$f_{5}, x_{0}=1.5$} \\
\hline N.M. & 7 & 2.154434690031883721759235 & $2.05 e^{-54}$ \\
\hline A.M. & 5 & 2.154434690031883721759235 & $-5.01 e^{-63}$ \\
\hline H.M. & 5 & 2.154434690031883721759235 & $-5.03 e^{-62}$ \\
\hline C.M. & 5 & 2.154434690031883721759235 & $-5.01 e^{-64}$ \\
\hline H.H.M. & 6 & 2.154434690031883721759293 & $7.86 e^{-27}$ \\
\hline NR.M. & 4 & 2.154434690031883721759292 & $8.98 e^{-24}$ \\
\hline Alg. 2.2 & 3 & 2.154434690031883721759294 & $2.00 e^{-27}$ \\
\hline Alg. 2.3 & 3 & 2.154434690031883721759293 & $1.33 e^{-50}$ \\
\hline \multicolumn{4}{|l|}{$f_{6}, x_{0}=3.5$} \\
\hline$N . M$. & 13 & 3.000000000000000000000000 & $1.52 e^{-48}$ \\
\hline A.M. & 7 & 3.000000000000000000000000 & $-4.32 e^{-48}$ \\
\hline H.M. & 8 & 3.000000000000000000000000 & $2.01 e^{-62}$ \\
\hline C.M. & 8 & 3.000000000000000000000000 & $2.05 e^{-62}$ \\
\hline H.H.M. & 12 & 3.000000000000000000000000 & $5.47 e^{-25}$ \\
\hline NR.M. & 6 & 3.00000010285594873990664 & $1.34 e^{-06}$ \\
\hline Alg. 2.2 & 4 & 3.00000000000000000000000 & $4.76 e^{-30}$ \\
\hline Alg. 2.3 & 4 & 3.00000000000000000000000 & $1.99 e^{-102}$ \\
\hline
\end{tabular}

\section{Conclusion}

We have established two new algorithms of fourth and fifth order convergence by using modified decomposition technique to coupled system. We have discussed convergence analysis of these newly established algorithms. Computational comparison of these algorithms has been made with some well known methods for solving nonlinear equations. From numerical table, we see that our new methods 
converge fast to the true solution of equations and are comparable with some classical methods.

\section{Competing Interests}

The author(s) do not have any competing interests in the manuscript.

\section{REFERENCES}

1. Frontini, M., \& Sormani, E. (2003). Some variant of Newtons method with third-order convergence. Applied Mathematics and Computation, 140(2), 419-426.

2. Adomain G. (1989). Nonlinear Atochastic Systems and Applications to Physics, Kluwer Academy Publishers. Dordrecht.

3. Chun, C. (2005). Iterative methods improving newton's method by the decomposition method. Computers \& Mathematics with Applications, 50(10), 1559-1568.

4. Noor, M. A., \& Noor, K. I. (2006). Some iterative schemes for nonlinear equations. Applied Mathematics and Computation, 183(2), 774-779.

5. Noor, M. A., Noor, K. I., Mohyud-Din, S. T., \& Shabbir, A. (2006). An iterative method with cubic convergence for nonlinear equations. Applied Mathematics and Computation, 183(2), 1249-1255.

6. Babolian, E., \& Biazar, J. (2002). On the order of convergence of Adomian method. Applied Mathematics and Computation, 130(2), 383-387.

7. Weerakoon, S., \& Fernando, T. G. I. (2000). A variant of Newton's method with accelerated third-order convergence. Applied Mathematics Letters, 13(8), 87-93.

8. Daftardar-Gejji, V., \& Jafari, H. (2006). An iterative method for solving nonlinear functional equations. Journal of Mathematical Analysis and Applications, 316(2), 753-763.

9. Saqib, M., Iqbal, M., Ali, S., \& Ismaeel, T. (2015). New Fourth and Fifth-Order Iterative Methods for Solving Nonlinear Equations. Applied Mathematics, 6(08), 1220-1227.

10. Frontini, M., \& Sormani, E. (2003). Modified Newton's method with third-order convergence and multiple roots. Journal of computational and applied mathematics, 156(2), 345-354.

11. Hasanov, V. I., Ivanov, I. G., \& Nedzhibov, G. (2002). A new modification of Newtons method. Appl. Math. Eng, 27, 278-286.

\section{Muhammad Saqib}

Department of Mathematics, Lahore Leads University, Lahore-54590, Pakistan.

e-mail: saqib270@yahoo.com

Muhammad Iqbal

Department of Mathematics, Lahore Leads University, Lahore-54590, Pakistan.

e-mail: iqbal66dn@yahoo.com 